\title{
A Dynamic Problem of Thermoelasticity
}

\author{
J. NEČnS and M. RÜŽiČKA
}

\begin{abstract}
A space-periodic problem of nonlinear thermoelasticity is considered. For an elastic. linear, isotropic, homogeneous, nonviscous body in small geometry, we obtain a nonlinear system of equations. For small coefficient of the heat extension a we find a time-global weak solution of the initial-value problem. The smallness of $a$ is independent of the length of the time interval and of the datas. The space periodicity of the solution is related to the absence of reflected waves. A mixed problem for a bounded domain. even with a smooth boundary, seems to be an open problem. Our work is closely related to that by J. Nečas [S] and by J. Nečas. A. Novotný and V. Sverák [6].
\end{abstract}

Key words: global solutions, a priori estimates

AMS subject classification: $73 \subset 25,73 \subset 50$

\section{Introduction}

Let $\Omega=(0,1)^{3} \subset \mathbf{R}^{3}$ be the body considered. We denote :

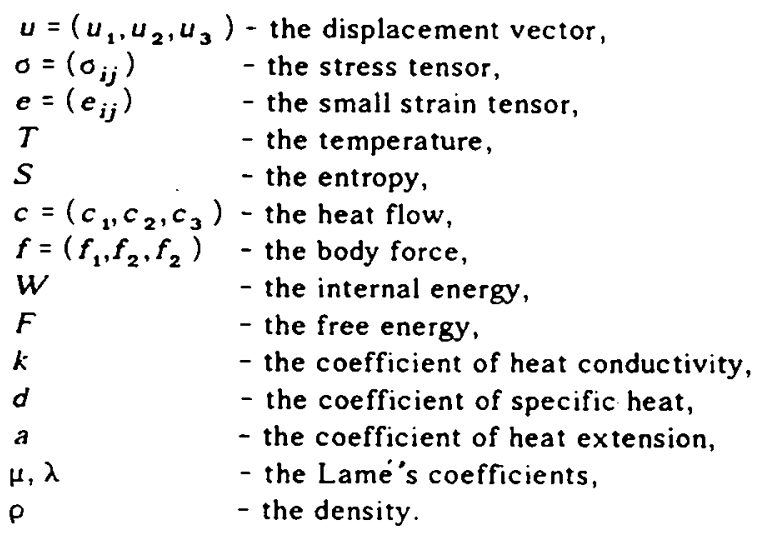

For the linear isotropic, homogeneous body, where we also take care of heat effects, we have the following equations:

$$
\begin{array}{ll}
\sigma_{i j}=\lambda \delta_{i j} e_{k k}+2 \mu e_{i j}-(3 \lambda+2 \mu) a \delta_{i j}\left(T-T_{0}\right) & \text { (Hooke 's law), } \\
\rho \ddot{u}_{i}=\partial \sigma_{i j} / \partial x_{j}+f_{i} & \text { (equation of motion), } \\
\dot{W}=\sigma_{i j} \dot{e}_{i j}-\partial c_{i} / \partial x_{i} & \text { (energy equation), }
\end{array}
$$

where $T_{0}$ is a constant and $\delta_{i j}$ denotes Kronecker's delta. For simplicity we suppose $\rho \equiv 1$. We use the summation convention over repeated indices. Let us assume that the free 
energy is only a function of the temperature and of the deformation, i.e. $F(e, T)=W(e, S)$

- TS. From this, by some calculations, we get

$$
\dot{W}=\sigma_{i j} \dot{e}_{i j}+d \dot{T}-(3 \lambda+2 \mu) a \dot{e}_{k k} T .
$$

If we use the equations $(0.3)$ and $(0.4)$ and the relation

$$
c_{i}(T, \nabla T)=-k(T, \nabla T) \partial T / \partial x_{i}
$$

we obtain

$$
\frac{\partial}{\partial x_{i}}\left(k \frac{\partial T}{\partial x_{i}}\right)=d \dot{T}+(3 \lambda+2 \mu) a \dot{e}_{k k} T
$$

Relation (0.5) is motivated by Fourier's law. The heat flow is subject to the principle of material frame indifference and so $k(T, \nabla T)$ has to be an isotropic function (see [9]). Further we require that $k$ is even with respect to $\left(T-T_{0}\right)$. The most simple function satisfying these properties is

$$
k(T, \nabla T)=a_{0}+a_{1}\left(T-T_{0}\right)^{2}+|\nabla T|^{2}, \text { with } a_{0}, a_{1}=\text { const } .
$$

For simplicity we set $a_{0}=a_{1}=1$. If we substitute the definition of the small strain tensor into $(0.1)$ and the result into $(0.2)$ we obtain

$$
\lambda \frac{\partial^{2} u_{j}}{\partial \lambda_{j} \partial x_{j}}+\mu\left(\frac{\partial^{2} u_{i}}{\partial x_{j} \partial x_{j}}+\frac{\partial^{2} u_{j}}{\partial x_{j} \partial x_{j}}\right)-(3 \lambda+2 \mu) a \frac{\partial T}{\partial \lambda_{j}}+f_{i}=\ddot{u}_{i}
$$

The system (0.6), (0.8) is a thermoelastic description of a linear, isotropic, homogeneous and thermoextensible body without viscosity. So we seek for the displacement vector $u$ and the temperature $T$, both defined on $Q_{I}=\Omega \times I$, with $I=\left(0, t_{0}\right)$ and satisfying $(0.6),(0.8)$.

By $\left(L^{p}(\Omega),\|\cdot\|_{p, \Omega}\right)$ and $\left(W_{p}^{m}(\Omega),\|\cdot\|_{m, p, \Omega}\right)$ we denote the Lebesgue and Sobolev spaces of space-periodic functions with period 1 . We put

$$
L^{P}\left(\Omega, \mathbf{R}^{3}\right)=L^{P}(\Omega) \times L^{P}(\Omega) \times L^{P}(\Omega), W_{P}^{m}\left(\Omega, R^{3}\right)=W_{p}^{m}(\Omega) \times W_{P}^{m}(\Omega) \times W_{P}^{m}(\Omega)
$$

with norms defined by the same symbols $\|\cdot\|_{p, \Omega}$ and $\|\cdot\|_{m, p, \Omega}$. Let $V$ be a Banach space. Then by $L^{P}(I, V)$ we denote the space of Bochner-measurable functions with values in $V$, for which $\int_{I}\|u(s)\|_{V}^{P} d s$ is finite. With the norm $\|u\|_{p, V}=\left(\int_{I}\|u(s)\|_{V}^{P}\right)^{1 / P}, L^{P}(I, V)$ is a Banach space. For details see [1]. Further we denote $u=\partial u / \partial t, \nabla u=\left(\partial u / \partial x_{1}, \partial u / \partial x_{2}\right.$, $\left.\partial u / \partial x_{3}\right), \nabla^{2} u=\left(\partial^{2} u / \partial x_{1}^{2}, \partial^{2} u / \partial x_{1} \partial x_{2}, \ldots \partial^{2} u / \partial x_{3}^{2}\right)$, weak convergence of a sequence $\left\{x_{n}\right\}$ to $x$ by $x_{n} \rightarrow x,(u, v)=\int_{\Omega} u(x) v(x) d x,\langle g, h\rangle=\int g(t) h(t) d t, Q_{t}=\Omega \times(0, t), t \in I$. At last, we will also denote by $c$ constants in various estimations.

\section{Existence of a solution}

From the above, we have to find functions $u: Q_{I} \rightarrow \mathbf{R}^{3}$ and $T: Q_{I} \rightarrow \mathbf{R}$ solving the problem

$$
\begin{aligned}
& (\lambda+\mu) \frac{\partial e_{k k}}{\partial x_{i}}+\mu \Delta u_{i}-\alpha \frac{\partial T}{\partial x_{j}}+f_{i}=\ddot{u}_{i}, \\
& \beta T+\alpha e_{k k} T-\frac{\partial}{\partial x_{j}}\left(k \frac{\partial T}{\partial x_{i}}\right)=0,
\end{aligned}
$$


where $\beta>0, \alpha \in\left(0, \alpha_{0}\right), \alpha_{0}=$ const and, according to $(0.7)$,

$$
k=1+\left(T-T_{0}\right)^{2}+|\nabla T|^{2}
$$

with initial conditions

$$
\begin{aligned}
& u(x, 0)=u_{0}(x) \text { and } u(x, 0)=u_{1}(x), x \in \Omega, \\
& T(x, 0)=T_{0}=\text { const. }>0, x \in \Omega .
\end{aligned}
$$

Further we will assume that

$$
u_{0} \in W_{2}^{2}\left(\Omega, \mathbb{R}^{3}\right), u_{0} \in W_{2}^{1}\left(\Omega, \mathbb{R}^{3}\right), f \in L^{2}\left(I, W_{2}^{1}\left(\Omega, \mathbf{R}^{3}\right)\right)
$$

Definition 1: A solution of the given problem (1.1) - (1.6) is a pair of functions $u: Q_{I} \rightarrow$ $\mathbf{R}^{3}$ and $T: Q_{I} \rightarrow \mathbf{R}$ satisfying the following conditions:

(i) $u \in L^{2}\left(I, W_{2}^{1}\left(\Omega, \mathbf{R}^{3}\right)\right), u \in L^{\infty}\left(I, L_{2}\left(\Omega, \mathbf{R}^{3}\right)\right), \ddot{u} \in L^{2}\left(I,\left(W_{2}^{1}\left(\Omega, \mathbf{R}^{3}\right)\right)^{\circ}\right)$,

$$
T \in L^{4}\left(I, W_{4}^{1}(\Omega)\right) \cap L^{2}\left(I, W_{2}^{2}(\Omega)\right), \dot{T} \in L^{4 / 3}\left(I, W_{4}^{1}(\Omega)\right) \text {. }
$$

(ii) $(\lambda+\mu) \frac{\partial e_{k k}}{\partial x_{j}}(x, t)+\mu \Delta u_{j}(x, t)-\alpha \frac{\partial T}{\partial x_{i}}(x, t)+f_{j}(x, e)=\ddot{u}(x, t)$ a.e. in $Q_{I}$.

(iii) $\int_{\Omega}\left(\beta \dot{T}(x, t) v(x)+\alpha \dot{e}_{k k}(x, t) T(x, t) v(x)\right.$

$$
\left.+k \frac{\partial T}{\partial x_{i}} \cdot(x, t) \frac{\partial v}{\partial x_{i}}(x)\right) d x=0 \quad \forall v \in W_{+}^{1}(\Omega) \text {, a.e. } t \in J .
$$

First, let us consider the following problem:

(P1) Let $T_{1} \in L^{4}\left(I, W_{4}^{1}(\Omega)\right)$. We seek for a weak solution $u$ of the problem (1.1), (1.4) for fixed $T \cong T_{1}$, i.e. we look for such function $u: Q_{I} \rightarrow \mathbf{R}^{3}$ that

(i) $u \in L^{\infty}\left(I, W_{2}^{1}\left(\Omega, \mathbf{R}^{3}\right)\right)$, $\dot{i} \in L^{\infty}\left(I, L_{2}\left(\Omega, \mathbf{R}^{3}\right)\right), \ddot{u} \in L^{2}\left(I,\left(W_{2}^{1}\left(\Omega, \mathbf{R}^{3}\right)\right)^{\prime}\right)$;

(ii) $\int_{\Omega}\left((\lambda+\mu) \frac{\partial u_{i}}{\partial x_{j}}(x, t) \frac{\partial v_{i}}{\partial x_{j}}(x)+\mu \frac{\partial u_{i}}{\partial x_{j}}(x, t) \frac{\partial v_{i}}{\partial x_{j}}(x)+\ddot{u}_{i}(x, t) v_{i}(x)\right) d x$

$$
=\int_{\Omega}\left(f_{i}(x, t) v_{j}(x)-\alpha \frac{\partial T_{1}}{\partial x_{i}}(x, t) v_{i}(x)\right) d x \quad \forall v \in W_{2}^{1}\left(\Omega, R^{3}\right) \text {, a.e. } t \in I .
$$

Lemma 1: There exists a unique weak solution u of Problem (P1), for which the following estimate holds :

$$
\begin{aligned}
& \int\left(|u(x, t)|^{2}+|\nabla u(x, t)|^{2}+|u(x, t)|^{2}\right) d x \\
& \quad \leq c\left(\left\|u_{0}\right\|_{1,2, \Omega}^{2}+\left\|u_{1}\right\|_{0,2, \Omega}^{2}+\|f\|_{2, O_{I}}^{2}+\alpha\left\|\nabla T_{1}\right\|_{2, O_{I}}^{2}\right) .
\end{aligned}
$$

Proof: The classical Galerkin method gives the existence of the weak solution of this linear problem (se, e.g., [8])

Theorem 1: Let $T_{1} \in L^{2}\left(I, W_{2}{ }^{2}(\Omega)\right) \cap L^{4}\left(I, W_{4}{ }^{1}(\Omega)\right)$. Then for the weak solution $u$ of the Problem (P1) we obtain 


$$
u \in L^{\infty}\left(I, W_{2}^{2}\left(\Omega, \mathbf{R}^{3}\right)\right), \quad \dot{u} \in L^{\infty}\left(I, W_{2}^{1}\left(\Omega, \mathbf{R}^{3}\right)\right), \ddot{u} \in L^{2}\left(I, L^{2}\left(\Omega, \mathbf{R}^{3}\right)\right)
$$
and

$$
\begin{aligned}
& \int\left(|\nabla \dot{u}(x, t)|^{2}+\left|\nabla^{2} u(x, t)\right|^{2}\right) d x \\
& \quad s c\left(\left\|u_{0}\right\|_{2,2, \Omega}^{2}+\left\|u_{1}\right\|_{1,2, \Omega}^{2}+\|\nabla f\|_{2, O_{1}}^{2}+\alpha\left\|\nabla^{2} T_{1}\right\|_{2, O_{1}}^{2}\right) \text { for a.e. } t \in I .
\end{aligned}
$$

Proof: We again use the Galerkin method. Let $\left\{g^{K}\right\}=\left\{\left(g_{1}^{K}, g_{2}^{K}, g_{3}^{K}\right)\right\}$ be a base of the space $W_{2}^{2}\left(\Omega, R^{3}\right)$ satisfying $\Delta g^{K}=-\lambda_{K} g^{K}$ for all $K \in N$. We define

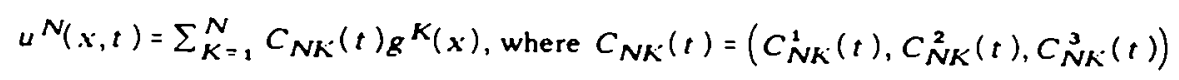

are defined by

$$
\begin{aligned}
& \int_{\Omega}\left((\lambda+\mu) \frac{\partial^{2} u_{j}^{N}}{\partial x_{j} \partial x_{j}}(x, t) \Delta g_{i}^{K}(x)+\mu \Delta u_{i}^{N}(x, t) \Delta g_{i}^{K}(x)-\ddot{u}_{i}^{N}(x, t) \Delta g_{i}^{K}(x)\right) d x \\
& \quad=\int_{\Omega}\left(-f_{i}(x, t) \Delta g_{i}^{K}(x)+\alpha \frac{\partial T_{1}}{\partial x_{i}}(x, t) \Delta g_{i}^{K}(x)\right) d x \quad \forall t \in I, \\
& C_{N K}(0)=\left(u_{0}, g^{K}\right), C_{N K}(0)=\left(u_{1}, g^{K}\right) .
\end{aligned}
$$

Multiplying the $K$-equation of (1.8) by $\dot{C}_{N K}(t)$, summing up over $K$ and integrating over $(0, t)$, we obtain

$$
\begin{aligned}
& \int\left(\mid \nabla \dot{\Omega} N_{\left.\left.(x, t)\right|^{2}+\left|\nabla^{2} u N(x, t)\right|^{2}\right) d x}\right. \\
& \quad \leq c\left(\left\|u_{0}(0)\right\|_{2,2, \Omega}^{2}+\left\|u_{1}(0)\right\|_{1,2, \Omega}^{2}+\|\nabla f\|_{2, Q_{I}}^{2}+\alpha\left\|\nabla^{2} T_{1}\right\|_{2, Q_{I}}^{2}\right) .
\end{aligned}
$$

From equations (1.7) and (1.9) we get that the sequence $\left\{u^{N}\right\}$ is bounded in $W_{2}^{2}\left(\Omega, R^{3}\right)$ and that the sequence $\left\{\dot{u}^{N}\right\}$ is bounded in $W_{2}^{1}\left(\Omega, R^{3}\right)$. Now, by a well-known technique we obtain our assertion

Let us for fixed $T_{1} \in L^{4}\left(I, W_{4}^{1}(\Omega)\right)$ and every $t \in I$ define the operator $A(t): W_{4}^{1}(\Omega) \rightarrow$ $\left(W_{t}^{1}(\Omega)\right)^{\prime}$ by

$$
(A(t) v, W)=\int_{\Omega}\left(\frac{1}{\beta}\left(1+\left(T_{1}-T_{0}\right)^{2}(x, t)+|\nabla v(x)|^{2}\right) \frac{\partial v}{\partial x_{j}}(x) \frac{\partial w}{\partial x_{j}}(x)\right) d x \quad \forall v, W \in W_{i}^{1}(\Omega) .
$$

Lemma 2: $A(t)$ is a monotonous, continuous and bounded operator (i.e. $A(t)$ maps bounded sets into bounded sets), which satisfies.

$$
\left(A(t)_{v}-A(t) w, v-w\right) \geq c \int_{\Omega}\left(|\nabla(v-w)(x)|^{2}+|\nabla(v-w)(x)| \cdot\right) d x \forall v, w \in W_{4}^{1}(\Omega) .
$$

Proof: Put $k_{i}(t, x, y)=\beta^{-1}\left(1+\left(T_{1}-T_{0}\right)^{2}(x, t)+|y|^{2}\right) y_{j}$. It is obvious that $k_{j}$ satisfies the Caratheodory condition and that the estimate $\left|k_{i}(t, x, y)\right| \leq c\left(g(x, t)+\sum_{i=1}^{3}\left|y_{i}\right|^{3}\right)$ holds for some function $g(\cdot, t) \in L^{* / 3}(\Omega)$. Now it is obvious (see [1:Lemma 1.6]) that $A(t)$ is a continuous and bounded operator. Further we have 


$$
\begin{aligned}
& \langle A(s) v-A(s) w, v-w\rangle \\
& \quad \geq \frac{1}{\bar{\beta}} \int_{\Omega}\left(\left(1+|\nabla v|^{2}\right) \frac{\partial v}{\partial \dot{x}_{i}}-\left(1+|\nabla w|^{2}\right) \frac{\partial w^{\prime}}{\partial x_{j}}\right) \frac{\partial(v-w)}{\partial x_{i}} d x \\
& \quad=\frac{1}{\bar{\beta}} \int_{\Omega} \int_{0}^{1}\left(1+3|\nabla w+t \nabla(v-w)|^{2}\right)(\nabla(v-w), \nabla(v-w)) d t d x \\
& \geq c\left(\int_{\Omega} \int_{0}^{1}|\nabla(v-w)|^{2}|\nabla w+t \nabla(v-w)|^{2} d t d x+\int_{\Omega}|\nabla(v-w)|^{2} d x\right) \\
& \quad \geq c \int_{\Omega}\left(|\nabla(v-w)|^{2}+|\nabla(v-w)|^{1}\right) d x
\end{aligned}
$$

Put $X^{*}=L^{4}\left(I, W_{4}^{1}(\Omega)\right)$. Then $X^{*}=L^{4 / 3}\left(I,\left(W_{1}^{1}(\Omega)\right)^{\circ}\right)$. Let us define the operator $A: X$ $\rightarrow \lambda "$ by

$$
(A v)(t)=A(t) v(t) \forall v \in X
$$

and the element $b \in X^{\prime \prime}$ by

$$
(b(t), v)=\frac{\alpha}{\beta} \int_{\Omega} \dot{u}_{j}(x, t)\left(\frac{\partial T_{1}}{\partial x_{j}}(x, t) v(x)+T_{1}(x, t) \frac{\partial v}{\partial x_{j}}(x)\right) d x \forall v \in W_{4}^{1}(\Omega),
$$

where $u$ is the corresponding solution of problem (P1) to $T_{1} \in X$. If we use this notation we can for (1.2), (1.3), (1.5) formulate the following problem:

(P2) Find $T_{2} \in \lambda$ satisfying

$$
\begin{aligned}
& \left(\dot{T}_{2}(t), v\right)+\left(A(t) T_{2}(t), v\right)=(b(t), v) \forall v \in W_{4}^{1}(\Omega), \text { a.e. } t \in I, \\
& T_{2}(x, 0)=T_{0} .
\end{aligned}
$$

Definition 2 : The function $T_{2} \in X$ is called a weak solution of the Problem (P2) if $T_{2}$ satisfies (1.10) and if $\dot{T}_{2} \in X^{\prime}$.

Theorem 2 : Let $T_{2} \in X$ and $u$ be the corresponding weak solution of the Problem (P1). Then there exists a weak solution $T_{2}$ of the Problem (P2).

Proof: Let us again use the Galerkin method. Let $\left\{g_{K}\right\}_{K=1}^{\infty}$ be a base of $W_{4}^{1}(\Omega)$. We define

$$
T_{2}^{N}(x, t)=\sum_{K=1}^{N} C_{N K}(t) g_{K}(x) \forall(x, t) \in Q_{I},
$$

where $C_{N K}$ are determined by

$$
\begin{aligned}
& \left(\dot{T}_{2}^{N}(t), g_{K}\right)+\left(A(t) T_{2}^{N}(t), g_{K}\right)=\left(b(t), g_{K}\right) \forall t \in I, \\
& C_{N K^{-}}(0)=\left(T_{0}, g_{K}\right) .
\end{aligned}
$$

From this we obtain

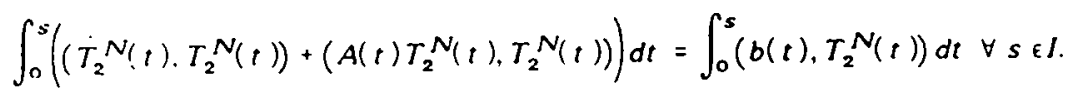


For the right - hand side we have in view of (1.7)

$$
\begin{aligned}
& \left|\int_{0}^{s} \int_{\Omega} u_{i}(x, t) \frac{\partial T_{1}}{\partial x_{i}}(x, t) T_{2}^{N}(x, t) d x d t\right| \\
& \quad s \int_{0}^{t}\left(\int_{\Omega}\left|\dot{u}_{i}(x, t)\right|^{2} d x\right)^{1 / 2}\left(\int_{\Omega}\left|\nabla T_{1}(x, t)\right|^{4} d x\right)^{1 / 4}\left(\int_{\Omega}\left|T_{2}^{N}(x, t)\right|^{4} d x\right)^{1 / 4} d t \\
& \quad s c\left(c_{1}+\alpha\left\|\nabla T_{1}\right\|_{2, Q_{I}}\right)\left\|\nabla T_{1}\right\|_{4}, Q_{I}\left(\int_{0}^{t_{0}}\left(\int_{\Omega}\left|T_{2}^{N}(x, t)\right|^{4} d x\right)^{1 / 3} d t\right)^{3 / 4} \\
& \quad s c_{0}\left(c_{1}+\alpha\left\|\nabla T_{1}\right\|_{2, Q_{I}}\right)\left\|\nabla T_{1}\right\|_{4, Q_{I}}\left(\left\|\nabla T_{2}^{N}\right\|_{2, Q_{I}}+\left\|T_{2}^{N}\right\|_{2, Q_{I}}\right) .
\end{aligned}
$$

where we have used in the last line the imbedding of $W_{2}{ }^{1}(\Omega)$ into $L^{\circ}(\Omega)$. In the same way we get

$$
\begin{aligned}
& \left|\int_{0}^{s} \int_{\Omega} \dot{u}_{i}(x, t) \frac{\partial T_{2}^{N}}{\partial x_{i}}(x, t) T_{1}(x, t) d x d t\right| \\
& \leq c_{0}\left(c_{1}+\alpha\left\|\nabla T_{1}\right\|_{2, Q_{I}}\right)\left\|\nabla T_{2}^{N}\right\|_{4}, Q_{I}\left(\left\|\nabla T_{1}\right\|_{2, Q_{I}}+\left\|T_{1}\right\|_{2, Q_{I}}\right) .
\end{aligned}
$$

From (1.12) we obtain, in view of (1.13), (1.14) and the monotonicity of $A(t)$,

$$
\begin{aligned}
& \int_{\Omega}\left|T_{2}^{N}(x, s)\right|^{2} d x+\int_{Q_{1}}\left(\left|\nabla T_{2}^{N}(x, t)\right|^{2}+\left|\nabla T_{2}^{N}(x, t)\right|^{4}\right) d x d t \\
& \quad s c\left(\left\|T_{2}^{N}(x, 0)\right\|_{2, \Omega}^{2}+\left(1+\left\|\nabla T_{1}\right\|_{2, Q_{I}}^{2}\right)\left(\left\|\nabla T_{1}\right\|_{4, Q_{I}}^{2}+\left\|T_{1}\right\|_{2, Q_{1}}^{2}\right)\right) .
\end{aligned}
$$

But

$$
\left(\int_{0}^{t_{0}}\left(\int_{\Omega} T^{2}(x, t) d x\right)^{2}+\int_{\Omega}|\nabla T(x, t)|^{4} d x d t\right)^{1 / 4}
$$

is an equivalent norm on the space $X$. And so there exist a weak convergent subsequence $\left\{T_{2}^{N}\right\} \subset X$ and elements $w \in X^{\prime}, z \in L^{2}(\Omega)$ such that

$$
\begin{aligned}
& T_{2}^{N} \rightarrow T_{2} \text { in } X, A T_{2}^{N} \rightarrow w \text { in } X^{\prime}, \\
& T_{2}^{N}\left(t_{0}\right) \rightarrow z \text { in } L^{2}(\Omega), T_{2}^{N}(0) \rightarrow T_{0} \text { in } W_{4}^{1}(\Omega),
\end{aligned}
$$

where we used the boundedness of $A$ and the imbedding of the set $\left\{T: T \in X, \dot{T} \in X^{\prime}\right\}$ into $C\left(I, L^{2}(\Omega)\right)$. By some calculation we obtain from (1.11)

$$
\dot{T}_{2} \in X, \dot{T}_{2}+w=b, T_{2}(0)=T_{0}, T_{2}\left(t_{0}\right)=z
$$

But

$$
\begin{aligned}
\varlimsup_{N \rightarrow \infty}\left\langle A T_{2}^{N}, T_{2}^{N}\right\rangle & =\varlimsup_{N \rightarrow \infty} 2^{-1}\left(\left\|T_{2}^{N}(0)\right\|_{2, \Omega}^{2}-\left\|T_{2}^{N}\left(t_{0}\right)\right\|_{2, \Omega}^{2}\right)+\left\langle b, T_{2}^{N}\right\rangle \\
& \leq 2^{-1}\left(\left\|T_{2}(0)\right\|_{2, \Omega}^{2}-\left\|T_{2}\left(t_{0}\right)\right\|_{2, \Omega}^{2}\right)+\left\langle b, T_{2}\right\rangle=\left\langle w, T_{2}\right\rangle .
\end{aligned}
$$

This, (1.15), the weak convergence of $T_{2}^{N}$ to $T_{2}$ in $X$ and the monotonicity of $A$ imply $A T_{2}=$ w. i.e. $T_{2}$ is a weak solution of the Problem (P2) 
Theorem 3: Let $T_{1} \in X \cap L^{2}\left(1, W_{2}^{2}(\Omega)\right)$. Then the weak solution $T_{2}$ of the Problem (P2) belongs to the space $L^{2}\left(I, W_{2}^{2}(\Omega)\right)$ and satisfies the estimate

$$
\left\|\nabla^{2} T_{2}\right\|_{2, Q_{1}}^{2} s c_{2}\left(c_{3}+\alpha\left(c_{s}+\alpha\left\|\nabla^{2} T_{1}\right\|_{2, Q_{I}}^{2}\right)+\left\|\nabla T_{2}\right\|_{4, Q_{I}}^{2}\left\|\nabla T_{2}\right\|_{4, Q_{I}}^{2}\right)
$$

Proof: (i) First we prove that $T_{2} \in L^{2}\left(I, W_{2}^{2}(\Omega)\right)$. For $v \in X$ and almost every $t \in I$ we have

$$
\begin{aligned}
\int_{\Omega}\left(\dot{T}_{2}(x, t) v(x, t)+\frac{1}{\beta}\left(1+\left(T_{1}+T_{0}\right)^{2}(x, t)+\left|\nabla T_{2}(x, t)\right|^{2}\right) \frac{\partial T_{2}}{\partial x_{i}}(x, t) \frac{\partial v}{\partial x_{i}}(x, t)\right) d x \\
\quad=-\int_{\Omega} \frac{\alpha}{\beta} T_{1}(x, t) \frac{\partial \dot{u}_{i}}{\partial x_{j}}(x, t) v(x, t) d x .
\end{aligned}
$$

We denote $\Delta_{\tau} x=\tau e^{l},|\tau|<h, h>0$ fixed, where $e^{l}(l=1,2,3)$ is a unit vector and $\left\{e^{1}, e^{2}, e^{3}\right\}$ is a base of $R^{3}$. Further we denote $\Delta_{\tau} w(x, t)=\tau^{-1}\left(w\left(x+\Delta_{\tau} x, t\right)-w(x, t)\right)$. From (1.17) we obtain

$$
\begin{aligned}
\int_{\Omega}\left\{\left(\dot{T}_{2}\left(x+\Delta_{\tau} x, t\right)-T_{2}(x, t)\right) v(x, t)\right. \\
+\frac{1}{\beta}\left[\left(1+\left(T_{1}-T_{0}\right)^{2}\left(x+\Delta_{\tau} x, t\right)+\left|\nabla T_{2}\left(x+\Delta_{\tau} x, t\right)\right|^{2}\right) \frac{\partial T_{2}}{\partial x_{i}}\left(x+\Delta_{\tau} x, t\right)\right. \\
\left.\left.\quad-\left(1+\left(T_{1}-T_{0}\right)^{2}(x, t)+\left|\nabla T_{2}(x, t)\right|^{2}\right) \frac{\partial T_{2}}{\partial x_{i}}(x, t)\right] \frac{\partial v}{\partial x_{i}}(x, t)\right\} d x \\
=-\int_{\Omega} \frac{\alpha}{\beta}\left[\frac{\partial \dot{u}_{j}}{\partial x_{j}}\left(x+\Delta_{\tau} x, t\right) T_{1}\left(x+\Delta_{\tau} x, t\right)-\frac{\partial \dot{u}_{i}}{\partial x_{i}}(x, t) T_{1}(x, t)\right] v(x, t) d x .
\end{aligned}
$$

In particular for $v(x, t)=\Delta_{\tau} T_{2}(x, t)$ we get

$$
\begin{aligned}
& \frac{1}{2} \int_{\Omega}\left(\left(\Delta_{\tau} T_{2}\right)^{2}(x, t)-\left(\Delta_{i} T_{2}\right)^{2}(x, 0)\right) d x+\frac{1}{\beta} \int_{Q_{I}}\left\{\frac{\partial \Delta_{\tau} T_{2}}{\partial x_{i}}(x, t) \frac{\partial \Delta_{\tau} T_{2}}{\partial x_{i}}(x, t)\right. \\
& +\frac{1}{2}\left(\left(T_{1}-T_{0}\right)^{2}\left(x+\Delta_{\tau} x, t\right)-\left(T_{1}-T_{0}\right)^{2}(x, t)\right) \frac{\partial \Delta_{\tau} T_{2}}{\partial x_{j}}(x, t) \frac{\partial \Delta_{\tau} T_{2}}{\partial x_{i}}(x, t) \\
& +\frac{1}{2}\left(\Delta_{\tau}\left(T_{1}-T_{0}\right)\right)^{2}(x, t) \frac{\partial \Delta_{\tau} T_{2}}{\partial x_{j}}(x, t)\left(\frac{\partial T_{2}}{\partial x_{j}}\left(x+\Delta_{\tau} x, t\right)+\frac{\partial T_{2}}{\partial x_{j}}(x, t)\right) \\
& +\frac{1}{2}\left(\left|\nabla T_{2}\left(x+\Delta_{\tau} x, t\right)\right|^{2}+\left|\nabla T_{2}(x, t)\right|^{2}\right) \frac{\partial \Delta_{\tau} T_{2}}{\partial x_{i}}(x, t) \frac{\partial \Delta_{\tau} T_{2}}{\partial x_{i}}(x, t) \\
& \left.+\frac{1}{2} \Delta_{\tau}\left|\nabla T_{2}(x, t)\right|^{2}\left(\frac{\partial T_{2}}{\partial x_{i}}\left(x+\Delta_{\tau} x, t\right)+\frac{\partial T_{2}}{\partial x_{j}}(x, t)\right) \frac{\partial \Delta_{\tau} T_{2}}{\partial x_{j}}(x, t)\right\} d x d t \\
& s \int_{O_{1}} \frac{\alpha}{\beta} \Delta_{\tau}\left(\frac{\partial \dot{u}_{i}}{\partial x_{i}}(x, t) T_{1}(x, t)\right) \Delta_{\tau} T_{2}(x, t) d x d t \\
& s c\left(\left(1+\left\|\nabla^{2} T_{1}\right\|_{2, Q_{I}}\right)\left\|T_{1}\right\|_{4} . O_{I}\left\|\Delta_{\tau} \Delta_{\tau} T_{2}\right\|_{4} . Q_{I}\right) \\
& \leq c\left(\left(1+\left\|\nabla^{2} T_{1}\right\|_{2, O_{I}}\right) \frac{1}{\varepsilon}\left\|T_{1}\right\|_{4, Q_{I}}^{2}+\varepsilon\left\|\partial \Delta T_{2} / \partial x_{e}\right\|_{4}^{2}, O_{I}\right) \text {, }
\end{aligned}
$$


where we used

$$
\begin{aligned}
& \int_{Q_{t}} \Delta_{\tau} u(x, t) \Delta_{\tau} T(x, t) d x d t=-\int_{Q_{t}} u(x, t) \Delta_{-\tau} \Delta_{\tau} T(x, t) d x d t \\
& \left\|\Delta_{-\tau} \Delta_{\tau} T\right\|_{4, \Omega} \leq\left\|\partial \Delta_{\tau} T / \partial x_{e}\right\|_{4}, O_{1}
\end{aligned}
$$

From this we get

$$
\begin{aligned}
& \int_{Q_{t}}\left\{\frac{\partial \Delta_{\tau} T_{2}}{\partial x_{i}}(x, t) \frac{\partial \Delta_{\tau} T_{2}}{\partial x_{i}}(x, t)\right. \\
&+\left(\left(T_{1}-T_{0}\right)^{2}\left(x+\Delta_{\tau} x, t\right)-\left(T_{1}-T_{0}\right)^{2}(x, t)\right) \frac{\partial \Delta_{\tau} T_{2}}{\partial x_{i}}(x, t) \frac{\partial \Delta_{\tau} T_{2}}{\partial x_{i}}(x, t) \\
&+\Delta_{\tau}\left(T_{1}-T_{0}\right)(x, t) \frac{\partial \Delta_{\tau} T_{2}}{\partial x_{j}}\left(\frac{\partial T_{2}}{\partial x_{i}}\left(x+\Delta_{\tau} x, t\right)+\frac{\partial T_{2}}{\partial x_{i}}(x, t)\right) \\
& \times\left(\left(T_{1}-T_{0}\right)\left(x+\Delta_{\tau} x, t\right)+\left(T_{1}-T_{0}\right)(x, t)\right) \\
&\left.+\left(\frac{\partial T_{2}}{\partial x_{j}}\left(x+\Delta_{\tau} x, t\right)+\frac{\partial T_{2}}{\partial x_{i}}(x, t)\right)^{2} \frac{\partial \Delta_{\tau} T_{2}}{\partial x_{j}}(x, t) \frac{\partial \Delta_{\tau} T_{2}}{\partial x_{i}}(x, t)\right\} d x d t \\
& s c\left(1+\left\|\nabla^{2} T_{1}\right\|_{2, Q_{I}}^{2}\left\|T_{1}\right\|_{4, Q_{I}}^{2}\right) .
\end{aligned}
$$

The third term on the left-hand side we carry to the right-hand side, where we use the estimate

$$
\begin{aligned}
& \int_{Q_{\tau}} \Delta_{\tau}\left(T_{1}-T_{0}\right)(x, t)\left(\frac{\partial T_{2}}{\partial x_{j}}\left(x+\Delta_{\tau} x, t\right)+\frac{\partial T_{2}}{\partial x_{j}}(x, t)\right) \\
& \quad \times \frac{\partial \Delta_{\tau} T_{2}}{\partial x_{i}}(x, t)\left(\left(T_{1}-T_{0}\right)\left(x+\Delta_{\tau} x, t\right)+\left(T_{1}-T_{0}\right)(x, t)\right) d x d t \\
& s c \frac{1}{\varepsilon}\left\|\Delta_{\tau} T_{1}\right\|_{4, Q_{I}}^{2}\left\|\nabla T_{2}\right\|_{4, Q_{I}}^{2}+c \varepsilon \int_{Q_{I}} \frac{\partial \Delta_{\tau} T_{2}}{\partial x_{j}}(x, t) \frac{\partial \Delta_{\tau} T_{2}}{\partial x_{j}}(x, t) \\
& \quad \times\left(\left(T_{1}-T_{0}\right)\left(x+\Delta_{\tau} x, t\right)+\left(T_{1}-T_{0}\right)(x, t)\right) d x d t
\end{aligned}
$$

for an $\varepsilon$ such that $c \varepsilon<1$. So from (1.18) we can obtain

$$
\begin{gathered}
\int_{Q_{I}} \frac{\partial \Delta_{\tau} T_{2}}{\partial x_{j}}(x, t) \frac{\partial \Delta_{\tau} T_{2}}{\partial x_{i}}(x, t) s c\left(1+\left\|\nabla^{2} T_{1}\right\|_{2, Q_{I}}^{2}\left\|T_{1}\right\|_{4, Q_{I}}^{2}\right) \\
\leq c\left(1+\left\|\nabla^{2} T_{1}\right\|_{2, Q_{I}}^{2}\left\|T_{1}\right\|_{4, Q_{I}}^{2}+\left\|\nabla T_{1}\right\|_{4, Q_{I}}^{2}\left\|\nabla T_{2}\right\|_{4, Q_{I}}^{2}\right) .
\end{gathered}
$$

We will use the following fact:

Let $u \in L^{P}(\Omega), 1<p<\infty$, let $\Delta_{\tau} u \in L^{P}\left(\Omega_{h}\right)$ for all $h>0,|\tau| \leq h$, and let $\left\|\Delta_{\boldsymbol{r}} u\right\|_{p, \Omega_{h}} \leq c_{1}<\infty$. Then, in sence of distributions, $\left\|\partial u / \partial x_{e} \mu\right\|_{p, \Omega_{h}} \leq c_{1}$.

Then we get from (1.19) that $T_{2} \in L^{2}\left(I, W_{2}^{2}(\Omega)\right)$.

(ii) Now we want to prove the estimate (1.16). We multiply (1.2) by $T_{2}^{\prime \prime}$, where $T_{2}{ }^{\prime \prime}$ is some second spatial derivative, and integrate over $Q_{l}$. Using partial integration we get 


$$
\begin{aligned}
\int_{\Omega} \frac{1}{2}( & \left.T_{2}^{\prime}\left(x, t_{0}\right)\right)^{2} d x-\int_{\Omega} \frac{1}{2}\left(T_{2}^{\prime}(x, 0)\right)^{2} d x \\
& +\int_{Q_{I}}\left(1+\left(T_{1}-T_{0}\right)^{2}(x, t)+\left|\nabla T_{2}(x, t)\right|^{2}\right) \frac{\partial T_{2}^{\prime}}{\partial x_{i}}(x, t) \frac{\partial T_{2}^{\prime}}{\partial x_{i}}(x, t) d x d t \\
= & \int_{O I}\left(\alpha T_{1}(x, t) \frac{\partial \dot{u}_{i}}{\partial x_{i}}(x, t) T_{2}^{\prime \prime}(x, t)\right. \\
& \left.-\left(1+\left(T_{2}-T_{0}\right)^{2}(x, t)+\left|\nabla T_{2}(x, t)\right|^{2}\right)^{\prime} \frac{\partial T_{2}}{\partial x_{i}}(x, t) \frac{\partial T_{2}^{\prime}}{\partial x_{i}}(x, t)\right) d x d t \\
= & \int_{Q_{I}}\left(\alpha T_{1}(x, t) \frac{\partial \dot{u}_{i}}{\partial x_{i}}(x, t) T_{2}^{\prime \prime}(x, t)-2\left(T_{1}-T_{0}\right)(x, t) T_{1}^{\prime}(x, t) \frac{\partial T_{2}}{\partial x_{i}}(x, t) \frac{\partial T_{2}^{\prime}}{\partial x_{i}}(x, t)\right. \\
& \left.-2 \frac{\partial T_{2}}{\partial x_{i}}(x, t) \frac{\partial T_{2}^{\prime}}{\partial x_{i}}(x, t) \frac{\partial T_{2}}{\partial x_{t}}(x, t) \frac{\partial T_{2}^{\prime}}{\partial x_{i}}(x, t)\right) d x d t .
\end{aligned}
$$

The last term on the right-hand side we carry to the left-hand side and so we get

$$
\begin{aligned}
& \int_{O_{1}}\left(\left|\nabla T_{2}^{\prime}(x, t)\right|^{2}+\left(T_{1}-T_{0}\right)^{2}(x, t)\left|\nabla T_{2}^{\prime}(x, t)\right|^{2}+\left|\nabla T_{2}(x, t)\right|^{2}\left|\nabla T_{2}^{\prime}(x, t)\right|^{2}\right) d x d t \\
& \quad s \int_{\Omega}\left|\nabla T_{2}(x, 0)\right|^{2} d x+\alpha\left|\int_{O_{I}} T_{1}(x, t) T_{2}^{\prime \prime}(x, t) \frac{\partial \dot{u}_{i}}{\partial x_{i}}(x, t) d x d t\right| \\
& \quad+2\left|\int_{Q_{I}}\left(T_{1}-T_{0}\right)(x, t) \frac{\partial T_{2}^{\prime}}{\partial x_{i}}(x, t) T_{1}^{\prime}(x, t) \frac{\partial T_{2}}{\partial x_{i}}(x, t) d x d t\right| \\
& \quad s c_{i}\left(c_{5}+\alpha\left\|T_{1} T_{2}^{\prime \prime}\right\|_{2, Q_{I}}\|\nabla i\|_{2, Q_{I}}+\left\|\left(T_{1}-T_{0}\right) \nabla T_{2}^{\prime}\right\|_{2, O_{I}}\left\|T_{1}^{\prime} \nabla T_{2}\right\|_{2, Q_{I}}\right) \\
& \quad \leq c_{0}\left(c_{5}+\alpha \varepsilon\left\|T_{1} T_{2}^{\prime \prime}\right\|_{2, Q_{I}}+\alpha \varepsilon^{-1}\left(c_{7}+\alpha\left\|\nabla^{2} T_{1}\right\|_{2, Q_{I}}\right)\right. \\
& \left.\quad+\varepsilon\left\|\left(T_{1}-T_{0}\right) \nabla T_{2}^{\prime}\right\|_{2, Q_{I}}^{2}+\varepsilon^{-1}\left\|T_{1}^{\prime} \nabla T_{2}\right\|_{2, Q_{I}}^{2}\right)
\end{aligned}
$$

for all $\varepsilon>0$. From this we obtain for some $\varepsilon$

$$
\int_{Q_{I}}\left|\nabla T_{2}^{\prime}(x, t)\right|^{2} d x d t \leq c_{8}\left(c_{s}+\alpha\left(c_{7}+\alpha\left\|\nabla^{2} T_{1}\right\|_{2, O_{I}}^{2}\right)+\left\|\nabla T_{1}\right\|_{4, O_{I}}^{2}\left\|\nabla T_{2}\right\|_{4, O_{I}}^{2}\right)
$$

Lemma 3 : Put, for some $R_{1}>0$,

$$
K_{1}=\left\{T, T \in X, \int_{Q_{I}}|\nabla T(x, t)|^{4} d x d t \leq R_{1}^{4}, \int_{Q_{I}} T^{4}(x, t) d x d t \leq R_{1}^{4}\right\} \text {. }
$$

Let $T_{1}, T_{2}$ denote the same functions as in Theorem 2. Then $B: T_{1} \rightarrow T_{2}$ is for $R_{1}$ large enough a mapping from $K_{1}$ into $K_{1}$.

Proof: Using the proof of Theorem 2, especially (1.13) - (1.15) we have, where all the norms are $L^{4}$ - norms, 


$$
\begin{aligned}
& \int_{Q_{1}}\left(T_{2}^{4}(x, t)+\mid \nabla T_{2}(x, t) \|^{4}\right) d x d t \\
& \quad s c \int_{0}^{t} 0\left\{\left(\int_{\Omega} T_{2}^{2}(x, t) d x\right)^{2}+\int_{\Omega} \mid \nabla T_{2}(x, t) \|^{4} d x\right\} d t \\
& \leq c_{9}\left(c_{10}+\alpha\left(c_{1}+\alpha\left\|\nabla T_{1}\right\|\right)\left(\left\|\nabla T_{1}\right\|\left\|\nabla T_{2}\right\|+\left\|\nabla T_{1}\right\|\left\|\nabla T_{2}\right\|+\left\|\nabla T_{2}\right\|\left\|\nabla T_{1}\right\|\right)\right) \\
& \leq c_{11}\left(c_{10}+\alpha c_{1}\left(\varepsilon^{-1}\left\|\nabla T_{1}\right\|^{2}+\varepsilon\left\|\nabla T_{2}\right\|^{2}+\varepsilon\left\|T_{2}\right\|^{2}+\varepsilon^{-1}\left\|T_{1}\right\|^{2}\right)\right. \\
& \left.\quad+\alpha^{2}\left(\varepsilon-1\left\|\nabla T_{1}\right\|^{3}+\varepsilon\left\|\nabla T_{2}\right\|^{2}+\left\|\nabla T_{1}\right\|^{2}+\varepsilon\left\|\nabla T_{2}\right\|^{4}+\varepsilon\left\|T_{2}\right\|^{4}+\left\|T_{1}\right\|^{3}\right)\right)
\end{aligned}
$$

for all $\varepsilon>0$. For some $\varepsilon$ we carry all the terms containing $T_{2}$ or $\nabla T_{2}$ on the left -hand side and so for $T_{1} \in K_{1}$ we have

$$
\begin{aligned}
& \int_{O_{1}}\left(T_{2}^{4}(x, t)+\left\|\nabla T_{2}(x, t)\right\|^{4}\right) d x d t \\
& \quad \leq c_{12}\left(c_{10}+\left\|\nabla T_{1}\right\|^{2}+\left\|T_{1}\right\|^{2}+\left\|\nabla T_{1}\right\|^{3}+\left\|T_{1}\right\|^{3}\right) \leq c_{12}\left(c_{10}+2 R^{2}+2 R^{3}\right),
\end{aligned}
$$

and the last term is smaller than $R_{1}^{4}$ for $R_{1}$ large enough

Lemma 4 : Put, for some $R_{2}>0$,

$$
K_{2}=\left\{T, T \in K_{1},\left\|\nabla^{2} T\right\|_{2, Q_{I}} \leq R_{2}, \dot{T} \in X^{\prime}\right\} .
$$

Let $T_{1}, T_{2}$ denote the same functions as in Theorem 3. Then $B: T_{1} \rightarrow T_{2}$, for $R_{2}$ large enough and $\alpha$ small enough but $\alpha$ independent of $R_{1}$ and $R_{2}$, is a mapping from $K_{2}$ into $K_{2}$.

Proof: For $T_{1} \in K_{2}$ we have (see (1.16))

$$
\begin{aligned}
\left\|\nabla T_{2}\right\|_{2, Q_{I}} \leq c_{2}\left(c_{3}+\alpha\left(c_{s}+\alpha\left\|\nabla^{2} T_{1}\right\|_{2, O_{I}}\right)+\left\|\nabla T_{1}\right\|_{\left.4, Q_{I}\left\|\nabla T_{2}\right\|_{4, Q_{I}}\right)}\right. \\
\leq c_{2}\left(c_{3}+\alpha c_{s}+\alpha^{2}\left\|\nabla^{2} T_{1}\right\|_{2, Q_{I}}^{2}+R_{1}^{4}\right) s c_{2}\left(c_{13}+\alpha^{2} R_{2}^{2}\right) .
\end{aligned}
$$

Now we take an $\alpha$ such that $c_{2} \alpha^{2}<1$, and so for $R_{2}$ large enough, $T_{2}$ is an element of $K_{2}$

Theorem 4 : Put

$$
K=\left\{T, T \in K_{2},\|\dot{T}\|_{X} \leq R_{3}\right\} .
$$

Then the mapping $B: T_{1} \rightarrow T_{2}$ has in $K$ a fixed point $T$, i.e. $T$ and the corresponding $u$ are a solution of (1.1) - (1.6).

Proof: We take an $R_{3}$ such that $\|\dot{T}\|_{X^{-}} \leq R_{3}$ for all $T_{1} \in K_{2} . K$ is a convex, closed and bounded subset of $\lambda$. So we have to show that $B$ is a weakly continuous operator, i.e. $\lambda_{n}$ $\rightarrow X$ in $X$ implies $B x_{n} \rightarrow B X$ in $X$. Let $\left\{T_{1}^{n}\right\} \subset K$ weakly converge to $T_{1}$. We denote $T_{2}^{n}$ $=B\left(T_{1}^{n}\right)$. From Lemma 4 we get that $\left\{T_{2}^{n}\right\} \subset K$. So there exists a $T_{3} \in K$ such that $T_{2}^{n} \rightarrow$ 
$T_{3}$ in $\lambda, \dot{T}_{2}^{\prime \prime} \rightarrow \dot{T}_{3}$ in $L^{\circ}\left(Q_{1}\right)$. From [2: Theorem 5.1] we get the strong convergence $T_{2}^{n} \rightarrow T_{3}$ and $T_{1}^{n} \rightarrow T_{1}$ in $L^{4}\left(Q_{t}\right)$. Further we have

$$
\begin{aligned}
& \int_{Q_{1}}\left(\dot{T}_{2}^{n}(x, t)-\dot{T}_{3}(x, t)\right)\left(T_{2}^{n}(x, t)-T_{3}(x, t)\right) d x d t \\
& \quad+\int_{Q_{1}} \frac{1}{\beta}\left[\left(1+\left(T_{1}^{n}-T_{0}\right)^{2}(x, t)+\left|\nabla T_{2}^{n}(x, t)\right|^{2}\right) \frac{\partial T_{2}}{\partial x_{i}}(x, t)\right. \\
& \left.\quad-\left(1+\left(T_{1}^{n}-T_{0}\right)^{2}(x, t)+\left|\nabla T_{3}(x, t)\right|^{2}\right) \frac{\partial T_{3}}{\partial x_{i}}(x, t)\right] \frac{\partial T_{2}^{n}-T_{3}}{\partial x_{i}}(x, t) d x d t \\
& =-\frac{\alpha}{\beta} \int_{O_{1}} \frac{\partial \dot{u}_{i}^{n}}{\partial x_{i}}(x, t) T_{1}^{n}(x, t)\left(T_{2}^{n}(x, t)-T_{3}(x, t)\right) d x d t \\
& \quad-\int_{Q_{I}} \dot{T}_{3}(x, t)\left(T_{2}^{n}(x, t)-T_{3}(x, t)\right) d x d t \\
& \left.-\frac{1}{\beta} \int_{O_{I}}\left(1+T_{1}^{n}(x, t)+\left|\nabla T_{3}(x, t)\right|^{2}\right) \frac{\partial T_{3}}{\partial x_{i}}(x, t)\right] \frac{\partial T_{2}^{n}-T_{3}}{\partial x_{i}}(x, t) d x d t
\end{aligned}
$$

The first term on the right - hand side we can estimate by

$$
c\left\|\nabla u^{n}\right\|_{2, Q}\left\|T_{2}^{n}\right\|_{4, Q_{I}}\left\|T_{2}^{n}-T_{3}\right\|_{4, Q I}
$$

which converges to zero in view of the boundedness of $\left\{T_{1}^{n}\right\}$ and $\left\{\nabla \dot{u}^{n}\right\}$. The second term on the right-hand side also converges to zero, because $\dot{T}_{3}$ is an element of $X^{\prime \prime}$. Also the third term on the right -hand side converges to zero, because $\partial T_{2}^{n} / \partial x_{i} \rightarrow \partial T_{3} / \partial x_{i}$ in $L^{4}\left(Q_{I}\right)$ and $\left(1+\left(T_{2}^{n}-T_{0}\right)^{2}(x, t)+|\nabla T(x, t)|^{2}\right) \partial T_{3} / \partial x_{i}(x, t)$ converges strongly in $L^{4 / 3}\left(Q_{I}\right)$. The left - hand side we can estimate from below by

$$
\begin{aligned}
\int_{\Omega}( & \left.T_{2}^{n}\left(x, t_{0}\right)-T_{3}\left(x, t_{0}\right)\right)^{2} d x-\int_{\Omega}\left(T_{2}^{n}(x, 0)-T_{3}(x, 0)\right)^{2} d x \\
& +\left\|\nabla\left(T_{2}^{n}-T_{3}\right)\right\|_{2, Q_{I}}^{2}+\left\|\nabla\left(T_{2}^{n}-T_{3}\right)\right\|_{4, Q_{I}}^{4} .
\end{aligned}
$$

We carry the second term on the other side, but also this term converges to zero. Thus we get that $\left\|T_{2}^{n}-T_{3}\right\|_{X} \rightarrow 0$. In view of the strong convergence of $T_{2}^{n}$ in $X$ we can tend with $n \rightarrow \infty$ in the equation

$$
\begin{aligned}
& \int_{Q_{1}} T_{2}^{n}(x, t) \varphi(x, t) d x d t \\
& \quad+\frac{1}{\beta} \int_{Q_{I}}\left(1+T_{1}^{n}(x, t)^{2}+\left|\nabla T_{2}^{n}(x, t)\right|^{2}\right) \frac{\partial T_{2}^{n}}{\partial x_{i}}(x, t) \frac{\partial \varphi}{\partial x_{i}}(x, t) d x d t \\
& =-\frac{\alpha}{\beta} \int_{O_{1}} \frac{\partial \dot{u}_{i}^{n}}{\partial x_{i}}(x, t) T_{1}^{4}(x, t) \varphi(x, t) d x d t \quad \forall \varphi \in X .
\end{aligned}
$$

So we get $B\left(T_{1}\right)=T_{3}$. Altogether we have the weak convergence of $T_{2}^{n}$ to $B\left(T_{1}\right)$ in $K$, i.e. weak continuity of $B$. By [7: Corollary 9.3] we get our assertion 


\section{REFERENCES}

[1] GAJEWSKI, H., GRÖGER, K., and K. ZnChaRIAS : Nichtlineare Operatorgleichungen und Operatordifferentialgleichungen. Berlin: Akademie - Verlag 1974.

[2] LIONS, J.L.: Ouelques méthodes de résolution des problemes aux limites non linéaires. Paris : Dunod Gauthier-Villars 1969.

[3] MaIS, G.E.: Teorija i zadačj mechaniky sploš nych sred (in Russian). Moskva:Mir 1974

[4] NEČAS, J.: Introduction to the Theory of Nonlinear Elliptic Equations (TeubnerTexte zur Mathemotik: Vol. 52). Leipzig: B.C. Teubner Verlagsges. 1983.

[5] NEÇAS, J.: Dynamic in the nonlinegr thermo-visco-elasticiry (to appear).

[6] NEČAs, J., NOVOTNY, A., and V. SVERAK: On the unjqueness of the solutid e solution to the

[7] ZEIDic of the nonlinear thermo-visco-elasticity. Math. Nachr. (to appear). (Teubner-Texte zur Mathematik. Vol. 2). (Teubner-Texte zur Mathematik: Vol. 2). Leipzig: B.G. Teubner Verlagsges. 1976.

[8] ZEIDLER. E.: Vorlesungon über nichtlineare Funktionalanalysis. II : Monofone Operatoren (Teubner-Texte zur Mathematik: Vol. 9). Leipzig: B.G. Teubner Verlagsges.
1976.

[9] ZEIDLER, E. : Nonlinear Functional Analysis and its Application. IV: Application to Marhematical Physics. Berlin - Heidelberg - Now York: Springer - Verlag 1988.

Received 16.08.1989; in revised form 25.01.1990

Authors' addresses:

Prof. Dr. Jindřch Nečas

Faculty of Mathematics and Physics

Dr. Michael Rížička

Charles University

Sokolovské 83

ČSSR - 18600 Praha 8

Sections of Mathematics

Karl - Marx - University

Karl - Marx - Platz

D(Ost) - 7010 Leipzig 